\title{
ON THE GRAPH OF DIVISIBILITY OF AN INTEGRAL DOMAIN
}

\author{
JASON GREENE BOYNTON AND JIM COYKENDALL
}

\begin{abstract}
It is well-known that the factorization properties of a domain are reflected in the structure of its group of divisibility. The main theme of this paper is to introduce a topological/graph-theoretic point of view to the current understanding of factorization in integral domains. We also show that connectedness properties in the graph and topological space give rise to a generalization of atomicity.
\end{abstract}

\section{INTRODUCTION}

Let $D$ be an integral domain with field of fractions $K$. Then, the group of divisibility $G(D)$ is defined to be the partially ordered additive group of principal fractional ideals with $a D \leqslant b D$ if and only if $a D \supseteq b D$. If $K^{\times}$is the multiplicative group of $K$ and if $U(D)$ is the group of units of $D$, then $G(D)$ is order isomorphic to the quotient group $K^{\times} / U(D)$ with the ordering $a U(D) \leqslant b U(D)$ if and only if $\frac{b}{a} \in D$.

It is well-known that the factorization properties of a domain are reflected in the structure of its group of divisibility. For example, an integral domain is a unique factorization domain if and only if its group of divisibility is a direct sum of copies of $\mathbb{Z}$ equipped with the usual product order. It is also true that the group of divisibility reflects more than just factorization properties of a domain. Indeed, it is not hard to check that a domain is a valuation domain if and only if its group of divisibility is totally ordered. We refer the interested reader to [6] for an excellent survey of material regarding the group of divisibility.

In 1968, Cohn introduced the notion of an atomic integral domain in 4. These are the domains in which every nonzero nonunit admits a finite factorization into irreducible elements. For several years, it was believed to be the case that atomicity in an integral domain was equivalent to the ascending chain condition on principal ideals (ACCP). However, in 1974, Anne Grams demonstrated that an atomic domain need not satisfy ACCP in [5. Grams was able to understand the subtle difference between atomicity and ACCP using the group of divisibility. Ten years later, Zaks added two more examples of an atomic domain without ACCP in [7. However, examples of atomic domains without ACCP are still relatively scarce.

The main theme of this work is to introduce a topological/graph-theoretic point of view to the current understanding of factorization in integral domains. That is, we find a graphical representation of the group of divisibility in order to detect various well-studied factorization properties of an integral domain. The contents of this paper is organized as follows. In Section 2, we recall a topological structure

2010 Mathematics Subject Classification. Primary 13F15; Secondary 13A05.

Key words and phrases. atomic, factorization, divisibility. 
that is naturally associated to a partially ordered set. In addition, we make the relevant graph-theoretic definitions needed in the sequel. In Section 3, we introduce the graph of divisibility of an integral domain and show that this graph detects the standard factorization properties studied in [1]. In Section 4, we examine the connectedness properties of the graph of divisibility using some elementary topology to do so. In Section 5, we will see that a connected graph of divisibility gives rise to a generalized atomicity. We also provide some examples in order to illustrate these notions.

\section{Some Definitions And Background}

In this section, we make some relevant definitions from graph theory and topology that will be used throughout. We refer the reader to 22 for a survey of known results about the Alexandrov topology.

Definition 2.1. Let $(X, \tau)$ be a topological space with neighborhood base $\mathcal{U}(x)=$ $\{U \in \tau: x \in U\}$.

(1) $(X, \tau)$ is called an Alexandrov space if arbitrary intersections of open sets remain open.

(2) For every $x$ in an Alexandrov space $X$, we set $M(x)=\cap_{U \in \mathcal{U}(x)} U$. The set $M(x)$ is called the minimal open set containing $x$.

Theorem 2.2. Let $(X, \tau)$ be an Alexandrov space.

(1) The collection of minimal open sets $\mathcal{N}=\{M(x): x \in X\}$ is a basis for the space $(X, \tau)$.

(2) $(X, \tau)$ is a $T_{0}$ space if and only if $M(x)=M(y) \Rightarrow x=y$.

(3) $(X, \tau)$ is (path and chain) connected if and only if for any pair of points $a, b \in X$, there exists a finite set of points $\left\{a=x_{0}, x_{1}, \ldots, x_{n}=b\right\}$ such that $N\left(x_{i-1}\right) \cap N\left(x_{i}\right) \neq \varnothing, i \leqslant n$.

In some sense, a $T_{0}$ Alexandrov space is the most natural topological structure induced by a partially ordered set. Indeed, if $(X, \leqslant)$ is any partially ordered set, then the sets of the form $M(a)=\{x \in X: x \leqslant a\}$ constitute a basis for a $T_{0}$ Alexandrov space $(X, \tau)$. Conversely, if $(X, \tau)$ is a $T_{0}$ Alexandrov space, we can define a relation $\leqslant$ on $X$ given by $a \leqslant b$ if and only if $a \in M(b)$. More precisely, we have the following result found in 2

Theorem 2.3. There is an isomorphism between the category of $T_{0}$ Alexandrov spaces with continuous maps and the category of partially ordered sets with order preserving set maps.

Now, let $(X, \tau)$ be any $T_{0}$ Alexandrov space with minimal neighborhood base $\mathcal{M}=\{M(a): a \in X\}$. One can construct a directed acyclic graph $\mathcal{G}(\mathcal{V}, \mathcal{E})$ determined by the space $(X, \tau)$. The set $\mathcal{V}$ of vertices is taken to be the underlying set $X$. Define an edge $a \rightarrow b$ if and only if $M(a) \subsetneq M(b)$ and there is no minimal base element $M(c)$ properly between $M(a)$ and $M(b)$. The resulting construction is a simple (no parallel edges) directed acyclic graph (SDAG). We now make the graphical representation of the previous constructions precise.

Definition 2.4. Let $(X, \leqslant)$ be any partially ordered set and define intervals $(-\infty, b]=$ $\{x \in X: x \leqslant b\}$ and $[a, b]=\{x \in X: a \leqslant x \leqslant b\}$ 
(1) We write $(X, \tau(\leqslant))$ to denote the the Alexandrov topology generated by the minimal neighborhood base $\mathcal{M}=\{M(a): a \in X\}$ where $M(a)=(-\infty, a]$.

(2) We write $\mathcal{G}(X, \mathcal{E}(\leqslant))$ to denote the directed acyclic graph whose vertices are the elements of $X$ and edges $a \rightarrow b$ if and only if $a<b$ and $[a, b]=\{a, b\}$.

Definition 2.5. Let $\mathcal{V}$ be a nonempty set.

(1) A finite directed path is a sequence of edges $\left\{e_{1}, e_{2}, \ldots, e_{n}\right\} \subset \mathcal{E}$ where $e_{i}=$ $\left(v_{i-1}, v_{i}\right)$ for each $i \in\{1,2, \ldots, n\}$. A finite directed path in $\mathcal{G}(\mathcal{V}, \mathcal{E})$ is also denoted by

$$
v_{0} \rightarrow v_{1} \rightarrow v_{2} \rightarrow \ldots \rightarrow v_{n} .
$$

A directed graph is said to be acyclic if there does not exist a path $\left\{e_{1}, e_{2}, \ldots, e_{n}\right\} \subset$ $\mathcal{E}$ such that $v_{0}=v_{n}$.

(2) A finite weak path is a sequence of ordered pairs $\left\{e_{1}, e_{2}, \ldots, e_{n}\right\} \subset \mathcal{V} \times \mathcal{V}$ where $e_{i}=\left(v_{i-1}, v_{i}\right)$ and either $\left(v_{i-1}, v_{i}\right)$ or $\left(v_{i}, v_{i-1}\right) \in \mathcal{E}$. A finite directed path in $\mathcal{G}(\mathcal{V}, \mathcal{E})$ is also denoted by

$$
v_{0} \leftrightarrow v_{1} \leftrightarrow \ldots \leftrightarrow v_{n} .
$$

(3) A directed graph $\mathcal{G}(\mathcal{V}, \mathcal{E})$ is said to be weakly connected if for every pair of vertices $v, w \in \mathcal{V}$ there exists a finite weak path $\left\{e_{1}, e_{2}, \ldots, e_{n}\right\}$ such that $v_{0}=v$ and $v_{n}=w$.

\section{The Graph of Divisibility}

In this section, we introduce the graph of divisibility of an integral domain. We will see that this graph gives a picture of the group of divisibility and can be used to detect certain factorization properties of a domain. Although this graph does not detect all divisibility relations, it does detect enough of the divisibility relation to clearly differentiate atomicity and ACCP (for example). For the remainder of this article, we denote the set of irreducible elements (atoms) of $D$ by $\operatorname{Irr}(D)$ and the set of atomic elements (expressible as a finite product of atoms) is denoted by $\mathcal{F}(D)$.

Definition 3.1. Let $D$ be any integral domain with field of fractions $K$ and let $K^{\times}$denote its multiplicative group.

(1) We write $G(D)$ to denote the group of divisibility $K^{\times} / U(D)$ written additively. We write $G(D)^{+}$to denote the positive elements of $G(D)$.

(2) We write $\mathcal{P}(D)$ to denote the group of nonzero principal fractional ideals of $D$ partially ordered by inclusion. We write $\mathcal{P}(D)^{+}$to denote the nonzero nonunit principal integral ideals of $D$.

Recall that the ordering in $(G(D), \leqslant)$ is given by $\bar{a} \leqslant \bar{b}$ if and only if $\frac{b}{a} \in D$. It readily follows that $0 \leqslant \bar{a}$ if and only if $a \in D$. It is easy to check that there exists a reverse order group isomorphism $G(D) \rightarrow \mathcal{P}(D)$ given by $\bar{a} \mapsto a D$. With Definition 2.1 in hand, we define a partial ordering on the set $\mathcal{P}(D)$ and consider the structure of the associated topological space and directed acyclic graph. The following lemma is the basis for the remainder of our investigations.

Lemma 3.2. Define a relation $\prec$ on $\mathcal{P}$ given by $a \prec b$ if and only if $\frac{a}{b} \in \mathcal{F}(D)$.

(1) $(\mathcal{P}(D), \preceq)$ is a partially ordered set.

(2) $(\mathcal{P}(D), \tau(\preceq))$ is a $T_{0}$ Alexandrov space with neighborhood base given by the collection $\mathcal{M}(a)=\left\{x \in \mathcal{P}: \frac{x}{a} \in \mathcal{F}(D)\right\}$. 
(3) $\mathcal{G}(\mathcal{P}(D), \mathcal{E}(\preceq))$ is a directed acyclic graph with directed edges $a \rightarrow b$ if and only if $\frac{a}{b} \in \operatorname{Irr}(D)$.

Proof. (1) It is never the case that $a \prec a$ since $\frac{a}{a}$ is a unit, and hence is not a product of atoms. Similarly, it is impossible that both $a \prec b$ and $a \succeq b$ can occur. Finally, if $a \prec b$ and $b \prec c$, then $\frac{a}{b} \in \mathcal{F}(D)$ and $\frac{b}{c} \in \mathcal{F}(D)$. Since the set $\mathcal{F}(D)$ is multiplicatively closed, we have that $\frac{a}{b} \cdot \frac{b}{c}=\frac{a}{c} \in \mathcal{F}(D)$ so that $a \prec c$.

(2) Follows immediately from (1) and the definition of $\prec$.

(3) If $a \rightarrow b$, then $a \prec b$ and $[a, b]=\{a, b\}$. It follows that $\frac{a}{b}=\pi_{1} \cdots \pi_{n}$ where each $\pi_{i} \in \operatorname{Irr}(D)$. In other words, $[a, b]=\left\{a, \pi_{1} \cdots \pi_{n-1} a, \ldots, \pi_{1} a, b\right\}$ and the condition $[a, b]=\{a, b\}$ forces $b=\pi_{1} a$. Therefore, $\frac{a}{b} \in \operatorname{Irr}(D)$ as needed. Conversely, if $\frac{a}{b}=\pi \in \operatorname{Irr}(D)$, then it is certainly true that $a \prec b$ and it suffices to check that $[a, b]=\{a, b\}$. But if $a \prec x \prec b$, then $\frac{a}{x}=\pi_{1} \cdots \pi_{n}$ and $\frac{x}{b}=\varsigma_{1} \cdots \varsigma_{m}$ where each $\pi_{i}, \varsigma_{i} \in \mathcal{A}(D)$. But then $\pi=\pi_{1} \cdots \pi_{n} \varsigma_{1} \cdots \varsigma_{m}$ forcing (without loss of generality) $\pi=\pi_{1}$ with the remaining factors units. It follows that $x=b$ as needed.

With Lemma 3.2 in hand, we make the definition central to our study.

Definition 3.3. We call $\mathcal{G}(\mathcal{P}(D), \mathcal{E}(\preceq))$ the graph of divisibility of $D$. We might also refer to the subgraph $\mathcal{G}\left(\mathcal{P}(D)^{+}, \mathcal{E}(\preceq)\right)$ the graph of divisibility.

We illustrate this definition with a few easy examples.

Example 3.4. (1) Let $D$ be a one-dimensional Noetherian valuation domain. It is well-known that $D$ is a PID with a unique nonzero prime ideal. So the the elements of $\mathcal{P}(D)^{+}$can be enumerated by the positive integers. We write $\mathcal{P}(D)^{+}=\left\{\pi, \pi^{2}, \pi^{3}, \ldots\right\}$ where $\pi$ is a chosen generator of the unique maximal ideal. The graph of divisibility $\mathcal{G}(\mathcal{P}(D), \mathcal{E}(\preceq))$ is the (branchless) tree that looks like

$$
\ldots \rightarrow \pi^{2} \rightarrow \pi \rightarrow 1 \rightarrow \frac{1}{\pi} \rightarrow \frac{1}{\pi^{2}} \rightarrow \ldots
$$

Similarly, the subgraph $\mathcal{G}\left(\mathcal{P}(D)^{+}, \mathcal{E}(\preceq)\right)$ looks like

$$
\ldots \rightarrow \pi^{3} \rightarrow \pi^{2} \rightarrow \pi
$$

(2) Let $D$ be a one-dimensional nondiscrete valuation domain. For the sake of concreteness, we will say that the corresponding value group is $\mathbb{Q}$. In this example, there are no irreducible elements and hence no two elements of $\mathcal{P}(D)$ are adjacent. It follows that $\mathcal{G}(\mathcal{P}(D), \mathcal{E}(\preceq))$ is just the collection of vertices corresponding to $\mathcal{P}(D)$ with no edges whatsoever. In fact, the graph of divisibility of any antimatter domain (no irreducible elements) consists of vertices only. Topologically speaking, $(\mathcal{P}(D), \tau(\preceq))$ is totally disconnected. That is, given any $a \in \mathcal{P}(D)$ we have that $M(a)=\{a\}$. The same is certainly true for the subspace $\left(\mathcal{P}(D)^{+}, \tau(\preceq)\right)$ and the subgraph $\mathcal{G}\left(\mathcal{P}(D)^{+}, \mathcal{E}(\preceq)\right)$.

Recall that a sink in a directed graph is a vertex with arrows in but no arrows out. We have the following lemma.

Lemma 3.5. Let $D$ be an integral domain and let $\mathcal{G}\left(P(D)^{+}, \mathcal{E}(\preceq)\right)$ be the associated graph of divisibility. Then,an element $\pi \in D^{\bullet}$ is irreducible in $D$ if and only if the node $\pi$ is a sink in $\mathcal{G}\left(P(D)^{+}, \mathcal{E}(\preceq)\right)$. 
It is well known that $D$ is atomic if and only if every element of $G(D)^{+}$can be written as a sum of minimal positive elements. Similarly, $D$ is satisfies ACCP if and only if every descending sequence of elements in $G(D)^{+}$stabilizes. As with the group of divisibility, the graph of divisibility can be used to characterize the well-studied factorization domains. We close this section with the following result.

Theorem 3.6. Let $D$ be an integral domain and let $\mathcal{G}\left(\mathcal{P}(D)^{+}, \mathcal{E}(\preceq)\right)$ be the associated graph of divisibility.

(1) $D$ is atomic if and only if for every non unit element $a \in \mathcal{P}(D)^{+}$, there exists a (finite) path originating from $a$ that terminates at an atom.

(2) $D$ satisfies ACCP if and only if for every $a \in \mathcal{P}(D)^{+}$, every path originating from $a$ terminates at an atom.

(3) $D$ is a BFD if and only if for every $a \in \mathcal{P}(D)^{+}$, every path originating from $a$ terminates at an atom and there is an upper bound on the lengths of all such paths.

(4) $D$ is an FFD if and only if for every $a \in \mathcal{P}(D)^{+}$, every path originating from $a$ terminates at an atom and there are finitely many such paths.

(5) $D$ is an HFD if and only if for every $a \in \mathcal{P}(D)^{+}$, every path originating from $a$ terminates at an atom and all such paths are of the same length.

\section{Some Connectedness Properties}

In this section, we consider the connectedness of the graph of divisibility. To do this, we will examine the connectedness of the associated Alexandrov topology. We conclude the section with a few examples.

Theorem 4.1. Let $D$ be an integral domain. The following statements for $a, b \in$ $K^{\times}$are equivalent.

(1) There exist atoms $\pi_{i}, \xi_{i} \in \operatorname{Irr}(D)$ such that $\frac{a}{b}=\frac{\pi_{1} \cdots \pi_{n}}{\xi_{1} \cdots \xi_{m}}$.

(2) The points $a, b$ belong to the same connected component in the Alexandrov topology $(\mathcal{P}(D), \tau(\preceq))$.

(3) There is a finite weak path connecting $a$ to $b$ in the graph of divisibility $\mathcal{G}(\mathcal{P}(D), \mathcal{E}(\preceq))$.

Proof. $(1) \Rightarrow(2)$ Suppose there exist atoms $\pi_{i}, \xi_{i} \in \operatorname{Irr}(D)$ such that $\frac{a}{b}=\frac{\pi_{1} \cdots \pi_{n}}{\xi_{1} \cdots \xi_{m}}$. To show that $a, b$ belong to the same connected component, it suffices to show that $M(a) \cap M(b)$ is nonempty. To this end, note that

$$
a \xi_{1} \cdots \xi_{m}=c=b \pi_{1} \cdots \pi_{n}
$$

implies that $c \prec a$ because $\frac{c}{a}=\xi_{1} \cdots \xi_{m}$. Similarly, we have that $c \prec b$, from which it follows that $c \in M(a) \cap M(b)$ as needed.

$(2) \Rightarrow(3)$ If $a, b$ belong to the same connected component, then there exists a finite set of points $\left\{a=x_{0}, x_{1}, \ldots, x_{n}=b\right\}$ such that $M\left(x_{i-1}\right) \cap M\left(x_{i}\right) \neq \varnothing$ for all $i \in\{1,2 \ldots, n\}$. Hence, we can choose a $c_{i} \in M\left(x_{i-1}\right) \cap M\left(x_{i}\right)$ so that $\frac{c_{i}}{x_{i-1}}, \frac{c_{i}}{x_{i}} \in \mathcal{F}(D)$, say $\frac{c_{i}}{x_{i-1}}=\xi_{1} \cdots \xi_{m}$ and,$\frac{c_{i}}{x_{i}}=\pi_{1} \cdots \pi_{n}$ where $\pi_{i}, \xi_{i} \in \operatorname{Irr}(D)$. It follows that there are directed paths

$$
c_{i} \rightarrow\left(x_{i-1} \xi_{1} \cdots \xi_{m-1}\right) \rightarrow \ldots \rightarrow\left(x_{i-1} \xi_{1}\right) \rightarrow x_{i-1}
$$

and

$$
c_{i} \rightarrow\left(x_{i} \pi_{1} \cdots \pi_{n}\right) \rightarrow \ldots \rightarrow\left(x_{i} \pi_{1}\right) \rightarrow x_{i}
$$


Hence, there is a weak path

$$
x_{i-1} \leftarrow \ldots \leftarrow c_{i} \rightarrow \ldots \rightarrow x_{i}
$$

for all $i \in\{1,2 \ldots, n\}$, and so there is a weak path connecting $a$ to $b$.

$(3) \Rightarrow(1)$ Suppose that $a, b$ are distinct points in the $T_{0}$ Alexandrov space $(\mathcal{P}(D), \tau(\preceq$ )). Then there exists a finite weak path connecting $a$ to $b$ say

$$
a=x_{0} \leftrightarrow x_{1} \leftrightarrow \ldots \leftrightarrow x_{n}=b .
$$

Using induction on $n$, we suppose that the result is true for all $k<n$. It follows from the existence of the weak path $a=x_{0} \leftrightarrow x_{1} \leftrightarrow \ldots \leftrightarrow x_{n-1}$ that $\frac{a}{x_{n-1}}=\frac{\pi_{1} \cdots \pi_{n}}{\xi_{1} \cdots \xi_{m}}$ where $\pi_{i}, \xi_{i} \in \operatorname{Irr}(D)$. Now observe that either $x_{n-1} \rightarrow b$ or $b \rightarrow x_{n-1}$. If $x_{n-1} \rightarrow b$ then by definition, we have $\frac{x_{n-1}}{b}=\pi \in \operatorname{Irr}(D)$. It follows that $\frac{a}{b}=\frac{a}{x_{n-1}} \cdot \frac{x_{n-1}}{b}=\frac{\pi \pi_{1} \cdots \pi_{n}}{\xi_{1} \cdots \xi_{m}}$, and a similar argument handles the case.

If $\mathfrak{F}$ is the subgroup of $K^{\times}$generated by $\operatorname{Irr}(D)$, then we can relate the number of connected components of $\mathcal{G}(\mathcal{P}, \mathcal{E}(\preceq))$ with the order of the quotient group $K^{\times} / \mathfrak{F}$ (a homomorphic image of the group of divisibility $K^{\times} / U(D)$ ). We immediately get the following result.

Corollary 4.2. There are 1-1 correspondences between the elements of $K^{\times} / \mathfrak{F}$, the connected components of $\mathcal{G}(\mathcal{P}(D), \mathcal{E}(\preceq))$, and the connected components of $(\mathcal{P}(D), \tau(\preceq))$.

Example 4.3. Consider the classical construction $D=\mathbb{Z}+x \mathbb{Q}[x]$. It is wellknown that the irreducible elements of $D$ are the primes $p \in \mathbb{Z}$ and $\mathbb{Q}[x]$-irreducible polynomials of the form $\pm 1+x q(x)$ where $q(x) \in \mathbb{Q}[x]$ (see [3]). For each $a \in D$ let us write $a(x)=\left(a_{0}, a_{1}, a_{2}, \ldots\right)$ where $a_{0} \in \mathbb{Z}$ and $a_{i} \in \mathbb{Q}$ for all $i \geqslant 1$. As with power series representations, we define the order of $a$ to be the natural number $\operatorname{ord}(a)=\min \left\{i \in \mathbb{N}: a_{i} \neq 0\right\}$. It follows from 3 that $a(x) \in \mathcal{F}(D)$ if and only if $\operatorname{ord}(a)=0$. We will now show that two polynomials $a, b \in D$ belong to the same connected component of $\left(\mathcal{P}(D)^{+}, \tau(\preceq)\right)$ if and only if $\operatorname{ord}(a)=\operatorname{ord}(b)$. Indeed, write $a(x)=x^{e_{0}} \bar{a}(x)$ and $b(x)=x^{f_{0}} \bar{b}(x)$ where $\operatorname{ord}(\bar{a})=0=\operatorname{ord}(\bar{b})$ (allowing $\left.e_{0}=0=f_{0}\right)$. If $a(x)$ is connected to $b(x)$, then by there exist atoms $\pi_{i}, \xi_{i} \in \operatorname{Irr}(D)$ such that

$$
\frac{a(x)}{b(x)}=\frac{\pi_{1}(x) \cdots \pi_{n}(x)}{\xi_{1}(x) \cdots \xi_{m}(x)}
$$

We now have the equation

$$
x^{e_{0}} \bar{a}(x) \xi_{1}(x) \cdots \xi_{m}(x)=x^{f_{0}} \bar{b}(x) \pi_{1}(x) \cdots \pi_{n}(x)
$$

and one easily checks that

$$
e_{0}=\operatorname{ord}\left(x^{e_{0}}\right)=\operatorname{ord}\left(x^{e_{0}} \bar{a} \xi_{1} \cdots \xi_{m}\right)=\operatorname{ord}\left(x^{f_{0}} \bar{b} \pi_{1} \cdots \pi_{n}\right)=\operatorname{ord}\left(x^{f_{0}}\right)=f_{0}
$$

For the converse, suppose that $e_{0}=f_{0}$. Again, using the fact that $\operatorname{ord}(\bar{a})=0=$ $\operatorname{ord}(\bar{b})$ is equivalent to $\bar{a}, \bar{b} \in \mathcal{F}(D)$, we have the existence of atoms $\pi_{i}, \xi_{i} \in \operatorname{Irr}(D)$ such that

$$
\frac{\bar{a}(x)}{\bar{b}(x)}=\frac{\pi_{1}(x) \cdots \pi_{n}(x)}{\xi_{1}(x) \cdots \xi_{m}(x)} .
$$

On the other hand, $e_{0}=f_{0}$ implies

$$
\frac{\bar{a}(x)}{\bar{b}(x)}=\frac{x^{e_{0}} \bar{a}(x)}{x^{f_{0}} \bar{b}(x)}=\frac{a(x)}{b(x)} .
$$


It follows that the distinct connected components of $\left(\mathcal{P}(D)^{+}, \tau(\preceq)\right)$ are given by the set $\left\{\operatorname{Irr}(D)=[2],[x],\left[x^{2}\right], \ldots\right\}$. In other words, there is no weak path $x^{m} \leftrightarrow \ldots \leftrightarrow x^{n}$ in $\mathcal{G}\left(\mathcal{P}^{+}, \mathcal{E}(\preceq)\right)$ whenever $m \neq n$.

Example 4.4. Let $x, y$ be indeterminates over the field $\mathbb{F}_{2}$.

(1) Now let $X=\left\{x^{\alpha}: \alpha \in \mathbb{Q}^{+}\right\}$and $Z_{1}=\left\{\frac{y^{k}}{x^{\alpha}}: \alpha \in \mathbb{Q}^{+}, k \in \mathbb{Z}^{+}\right.$, and $k \geqslant 2\}$. We determine the number of connected components in the graph of divisibility of the domain $D_{1}=\mathbb{F}_{2}\left[X, y, Z_{1}\right]_{\left(X, y, Z_{1}\right)}$. To do this, we first observe that the integral closure of $D_{1}$ is the rank 2 valuation domain $V=\mathbb{F}_{2}[X, Z]_{(X, Z)}$ where $Z=\left\{\frac{y}{x^{\alpha}}: \alpha \in \mathbb{Q}^{+}\right\}$. The value group of $V$ is $\mathbb{Z} \oplus \mathbb{Q}$ ordered lexicographically and it is easy to check that every element of $V^{\bullet}$ is a unit multiple of $y^{k}$ or $x^{r} y^{k}$ where $(k, r) \in \mathbb{Z}^{+} \oplus \mathbb{Q}$. It is not hard to check that every element of $\operatorname{Irr}\left(D_{1}\right)$ has value $(1,0)$. It is now an easy matter to check that the connected components of $\mathcal{G}\left(\mathcal{P}\left(D_{1}\right)^{+}, \mathcal{E}(\preceq)\right)$ are given in terms of their values by

$$
\{[(0, \alpha)]\}_{\alpha \in \mathbb{Q}^{+}} \cup\{[(k, 0)]\}_{k \in \mathbb{Z}^{+}} \cup\{[(k, \alpha)]\}_{\substack{k \geqslant 2 \\ \alpha<0}} .
$$

For example, consider the elements $f=x^{\frac{1}{2}}$ and $g=\frac{y^{3}}{x^{\frac{1}{3}}}$. Then $v(f)=\left(0, \frac{1}{2}\right)$ and $v(g)=\left(3, \frac{1}{3}\right)$. Then $v\left(\frac{g}{f}\right)=v(g)-v(f)=\left(3,-\frac{1}{6}\right)$ cannot be written in the form $m(1,0)$ where $m \in \mathbb{Z}$. In other words, $\frac{g}{f}$ cannot be expressed as the quotient of atomic elements.

(2) If $Z_{2}=\left\{\frac{y^{k}}{x^{j}}: j \in \mathbb{Z}^{+}, k \in \mathbb{Z}^{+}\right.$, and $\left.k \geqslant 2\right\}$ and $D_{2}=\mathbb{F}_{2}\left[x, y, Z_{2}\right]_{\left(x, y, Z_{2}\right)}$, then $\left(\mathcal{P}\left(D_{2}\right)^{+}, \tau(\preceq)\right)$ is a connected Alexandrov space. Equivalently, the graph of divisibility $\mathcal{G}\left(\mathcal{P}\left(D_{2}\right)^{+}, \mathcal{E}(\preceq)\right)$ is weakly connected. One need only check that the integral closure of $D_{2}$ has the discrete value group $\mathbb{Z} \oplus \mathbb{Z}$ ordered lexicographically. Again, it is not hard to check that every element of $\operatorname{Irr}\left(D_{2}\right)$ has value $(0,1)$ or $(1,0)$ and given any $f, g \in D_{2}$, we have that $v\left(\frac{g}{f}\right)=m(1,0)+n(0,1)$ where $m, n \in \mathbb{Z}$.

\section{Some Generalizations of Atomicity}

In this section, we show that a connected graph of divisibility gives rise to a generalization of atomicity.

Definition 5.1. Let $D$ be any integral domain.

(1) $D$ is called almost atomic if for every $a \in D^{\bullet}$, there exist atoms $\left\{\pi_{i}\right\} \subset$ $\operatorname{Irr}(D)$ such that $a \pi_{1} \cdots \pi_{n} \in \mathcal{F}(D)$.

(2) $D$ is called quasi atomic if for every $a \in D^{\bullet}$, there exists an element $b \in D$ such that $a b \in \mathcal{F}(D)$.

It is easy to see that almost atomic implies quasi atomic. Also, if $D$ is quasi atomic, it is not hard to show that every nonzero prime ideal of $D$ contains an irreducible element. We have the following lemma.

Lemma 5.2. Given an integral domain $D$, each condition below implies the next:

(1) $D$ is atomic

(2) $D$ is almost atomic

(3) $D$ is quasi atomic

(4) Every nonzero prime ideal of $D$ contains an irreducible element. 
Proof. It suffices to show that (3) implies (4). Suppose that $D$ is quasi atomic. If $a$ is a nonzero element of a prime ideal $P$, then there is $b \in D$ such that $a b=\pi_{1} \cdots \pi_{n}$ where each $\pi_{i} \in \operatorname{Irr}(D)$. But then $\pi_{1} \cdots \pi_{n} \in P$ so that $\pi_{i} \in P$ for some $i \leqslant n$.

These observations give an example of an integral domain that is not quasi atomic.

Example 5.3. As in Example 4.3, let $D=\mathbb{Z}+x \mathbb{Q}[x]$. Then $x \mathbb{Q}[x]$ is a prime ideal of $D$ that contains no irreducible element. To see this, recall from 4.3 that if $f \in \operatorname{Irr}(D)$, then $\operatorname{ord}(f)=0$. But $f \in x \mathbb{Q}[x]$ if and only if $\operatorname{ord}(f) \geqslant 1$. It follows from Lemma 5.2 that $D$ is not quasi atomic.

We now show the connection between almost atomicity and a connected graph of divisibility.

Theorem 5.4. The following statements are equivalent for a domain $D$.

(1) $D$ is almost atomic.

(2) $(\mathcal{P}, \tau(\preceq))$ is connected.

(3) $\mathcal{G}(\mathcal{P}, \mathcal{E}(\preceq))$ is weakly connected.

Proof. $(1) \Rightarrow(2) \quad$ Choose any two points $a, b \in(\mathcal{P}, \tau(\preceq))$. If $D$ is almost atomic, there exist atoms $\pi_{i}, \xi_{i} \in \operatorname{Irr}(D)$ such that $a \pi_{1} \cdots \pi_{n}$ and $b \xi_{1} \cdots \xi_{m} \in \mathcal{F}(D)$. In other words, there exist atoms $\sigma_{i}, \varsigma_{i} \in \mathcal{A}(D)$ such that $\frac{a}{b}=\frac{\pi_{1} \cdots \pi_{n} \sigma_{i} \cdots \sigma_{j}}{\xi_{1} \cdots \xi_{m} \varsigma_{i} \cdots \varsigma_{k}}$. It follows from Theorem 4.1 that any pair of points in $(\mathcal{P}, \tau(\preceq))$ belong to the same connected component.

$(2) \Rightarrow(3)$ Follows immediately from Theorem 4.1.

$(3) \Rightarrow(1)$ Since $\mathcal{G}(\mathcal{P}, \mathcal{E}(\preceq))$ is weakly connected, there is a weak path connecting any $\frac{a}{1} \in \mathcal{P}$ (where $a \in D$ ) to an element of the form $\frac{\pi}{1}$ where $\pi \in \operatorname{Irr}(D)$. Theorem 4.1 implies that there exist atoms $\pi_{i}, \xi_{i} \in \operatorname{Irr}(D)$ such that $\frac{a}{\pi}=\frac{\pi_{1} \cdots \pi_{n}}{\xi_{1} \cdots \xi_{m}}$. In other words, there exist atoms $\xi_{i} \in \operatorname{Irr}(D)$ such that $a \xi_{1} \cdots \xi_{m} \in \mathcal{F}(D)$.

Using the Theorem 5.4 and the results from the previous section, we are led to an example of an almost atomic domain that is not atomic.

Example 5.5. (1) As in Example 4.4(1), let $D_{1}=\mathbb{F}_{2}\left[X, y, Z_{1}\right]_{\left(X, y, Z_{1}\right)}$ where $X=\left\{x^{\alpha}: \alpha \in \mathbb{Q}^{+}\right\}$and $Z_{1}=\left\{\frac{y^{k}}{x^{\alpha}}: \alpha \in \mathbb{Q}^{+}, k \in \mathbb{Z}^{+}\right.$, and $\left.k \geqslant 2\right\}$. Since the connected components are in a 1-1 correspondence with $\mathbb{Q}$, it is certainly not the case that $D_{1}$ is almost atomic (Theorem 5.4). However, it is the case that $D_{1}$ is quasi atomic. Indeed, given any $f \in D_{1}^{\bullet}$, we can write $v(f)=(k, \alpha)$. There is a $g \in D_{1}$ such that $v(g)=(2,-\alpha)$ and so

$$
v(f g)=v(f)+v(g)=(k+2,0)=(1,0)+\ldots+(1,0) .
$$

Translating this information back to $D_{1}$, we get that $f g=y^{k+2} u$ for some unit $u \in V$. Note that if $y^{n+1} u \in D_{1}$ for some unit $u \in V$, then $y^{n+1} u=$ $v_{1} x^{\alpha}+v_{2} \frac{y^{l}}{x^{\beta}}+v_{3} y$ where either $v_{i} \in U\left(D_{1}\right)$ or $v_{i}=0$. If $n>0$, then $v_{1}=0=v_{3}$. It follows that $l \geqslant n+1$ so that $y^{n} u=v_{2} \frac{y^{l-1}}{x^{\beta}}$. Therefore, $y u=v_{2} \frac{y^{l-n}}{x^{\beta}} \in D_{1}$ as $l-n \geqslant 1$. It follows from all of this that $f g \in \mathcal{F}(D)$ as needed.

(2) As in Example 4.4(2), let $D_{2}=\mathbb{F}_{2}\left[x, y, Z_{2}\right]_{\left(x, y, Z_{2}\right)}$ where $Z_{2}=\left\{\frac{y^{k}}{x^{j}}: j \in\right.$ $\mathbb{Z}^{+}, k \in \mathbb{Z}^{+}$, and $\left.k \geqslant 2\right\}$. Since $\mathcal{G}\left(\mathcal{P}\left(D_{2}\right)^{+}, \mathcal{E}(\preceq)\right)$ is weakly connected, it must be the case that $D_{2}$ is almost atomic. However, it is not atomic since, 
for example, $v\left(\frac{y^{2}}{x^{\frac{1}{2}}}\right)=\left(2, \frac{1}{2}\right)$ cannot be written as an $\mathbb{N}$-linear combination $m(1,0)+n(0,1)$.

Acknowledgements. The authors would like to thank the North Dakota State University Department of Mathematics for their continued support.

\section{REFERENCES}

[1] D.D. Anderson, D.F. Anderson, and M. Zafrullah. Factorization in integral domains. J. Pure Appl. Algebra, 69:1-19, 1990.

[2] F. G. Arenas. Alexandroff spaces. Acta Math. Univ. Comenianae, 68:17-25, 1999.

[3] S. T. Chapman and W. W. Smith. Restricted elasticity in rings of integer-valued polynomials determined by finite subsets. Monatsh. Math., 148:195-203, 2006.

[4] P. M. Cohn. Bézout rings and their subrings. Proc. Cambridge Philos. Soc., 75:321-329, 1974.

[5] Anne Grams. Atomic rings and the ascending chain condition for principal ideals. Proc. Cambridge Philos. Soc., 75:321-329, 1974.

[6] Joe L. Mott. The group of divisibility and its applications. In Conference on Commutative Algebra (Univ. Kansas, Lawrence, Kan., 1972), pages 194-208. Lecture Notes in Math., Vol. 311. Springer, Berlin, 1973.

[7] Abraham Zaks. Atomic rings without a.c.c. on principal ideals. J. Algebra, 74(1):223-231, 1982.

Department of Mathematics, North Dakota State University, Fargo, ND 58108

E-mail address, J. G. Coykendall: jason. boynton@ndsu.edu

Department of Mathematics, North Dakota State University, Fargo, ND 58108

E-mail address, J. Coykendall: jim.coykendall@ndsu.edu 